\title{
Detection Characteristics of Ge Detectors With Microstructured Amorphous Ge Contacts
}

\author{
D. Protić and T. Krings
}

\begin{abstract}
The established technique for producing position-sensitive structures by means of photolithography and subsequent plasma etching of grooves through detector contacts was applied for amorphous germanium contacts (a-Ge contacts). A simple 50-strip structure with a pitch of $615 \mu \mathrm{m}$ was produced. Charge sharing between two adjacent strips (33 $\mathrm{mm}$ length) was investigated with the aid of $60-\mathrm{keV}$ photons and 5.8-MeV $\alpha$-particles. No charge losses were detected when irradiating the structured a-Ge contact of the 16-mm-thick detector with 60-keV photons. The same was concluded for $\alpha$-particles hitting the unstructured $\mathrm{p}^{+}$-contact made by boron implantation. Moreover, excellent position information for $\alpha$-particles, with an accuracy of about $30 \mu \mathrm{m}$, could be extracted in this case. Surprising results were obtained for $\alpha$-particles impinging on the structured a-Ge contact. No coincident signals from the two adjacent strips caused by $\alpha$-particles were observed. But a normally positive signal from one of the strips occasionally coincided with a negative signal from the other strip and vice versa. Using a cooled FET assembly mounted near a strip, an energy resolution of $0.78 \mathrm{keV}$ [FWHM] for 60-keV photons was achieved and a slightly better resolution for lower energies. Even better energy resolutions will probably be possible, because the measuring setup was far from being optimized.
\end{abstract}

Index Terms-Amorphous germanium contacts (a-Ge contacts), charge splitting between the strips, germanium radiation detectors, microstrip germanium detectors, plasma etched grooves, position-sensitive germanium detectors, structured a-Ge contacts.

\section{INTRODUCTION}

$\mathbf{V}$ ERY recently, a new method for producing position sensitive structures on germanium detectors having amorphous Ge contacts (a-Ge contacts) was presented [1]. The method is based on the well-established technique for manufacturing position-sensitive germanium and silicon detectors by means of photolithography and subsequent plasma etching of grooves through implanted or thin lithium-diffused contacts [2]-[4].

Position-sensitive structures on a-Ge contacts realized with the aid of plasma-etched grooves obviously have several advantages compared to earlier reported techniques where the structures were created by metallic strips evaporated on a-Ge contacts through shadow masks [5], [6]. The gap between the metallic strips (200-500 $\mu \mathrm{m}$ in width) and the region below it represent a part of the detector where charge collection is incomplete [6]-[8], as shown by measurements using $60-\mathrm{keV}$ photons. Measurements with a position-sensitive germanium detector realized by $\sim 60 \mu \mathrm{m}$-wide plasma-etched grooves

Manuscript received November 14, 2003; revised February 3, 2004. The authors are with the Forschungszentrum Jülich $\mathrm{GmbH}$, Institut für Kernphysik, 52425 Jülich, Germany (e-mail: d.protic@fz-juelich.de).

Digital Object Identifier 10.1109/TNS.2004.829577 through the a-Ge contact [1] indicate that practically no energy losses were caused by the grooves. Unfortunately, these measurements were carried out with relatively high-energy photons $(661 \mathrm{keV})$. To compare the results with those obtained for structures fabricated by the evaporation of metallic strips on a-Ge contacts [6]-[8], one has to perform the measurements using $60-\mathrm{keV}$ photons irradiating the position-sensitive structure. Moreover, an even stronger proof of the influence of the grooves on charge collection would be possible by irradiating the adjacent strips with $5.8-\mathrm{MeV} \alpha$-particles. Their range of about $23 \mu \mathrm{m}$ matches the groove dimensions quite well ( $\sim 50 \mu \mathrm{m}$ in width and $\sim 20 \mu \mathrm{m}$ in depth). Valuable information about the charge collection efficiency in and across the groove itself can be expected.

Furthermore, microstructures defined with narrow grooves, even below $10 \mu \mathrm{m}$, can be easily fabricated. To our knowledge, the smallest width of the gap between metallic strips reported up to now was $200 \mu \mathrm{m}$ [9]. But making smaller gaps with the aid of photolithography should be feasible [10].

Another important aspect is how good an energy resolution can be achieved with the elements of the grooved structures. Due to the absence of germanium (relative dielectric constant $=$ 16) in the grooves, the interstrip capacitance could be considerably lower than in the structures without grooves (up to 50\% in our case) [11]. Moreover, for the same reason, the dielectric noise contribution [12] should be smaller in the grooved structures. Both effects should enable superior energy resolution for detector elements defined by grooves.

For this purpose, the first stage of a preamplifier should be placed very close to the detector and one of the strips connected by bonding to the cooled FET. The aim is to show that the detectors with grooved strip structures could be connected to the chip electronics, placed very close to the detector, which could provide excellent energy resolutions for low energy photons.

\section{DETECTOR}

For these investigations, the detector mentioned in [1] should be mounted into the new housing. But during the transfer, a part of the a-Ge contact and a region on the rim were damaged. To eliminate the damage, the outline of the contacts was changed by cutting the detector to a square of $47 \times 47 \mathrm{~mm}^{2}$ having slightly rounded corners and the a-Ge contact was removed by lapping.

The new a-Ge contact was prepared on the chemically etched surface by the evaporation of a $0.10-\mu \mathrm{m}$-thick amorphous Ge-layer subsequently covered by a $0.38-\mu \mathrm{m}$-thick layer of evaporated aluminum.

The thickness of the n-type germanium (estimated net impurity concentration of $7 \cdot 10^{9} \mathrm{~cm}^{-3}$ ) was practically unchanged 


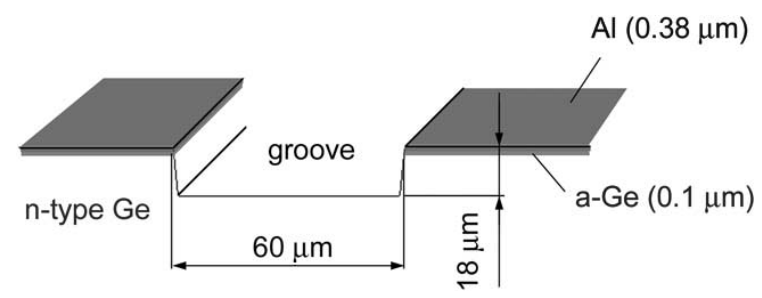

Fig. 1. Schematic view of the a-Ge contact divided into 50 strips with a pitch of $615 \mu \mathrm{m}$ by $18-\mu \mathrm{m}$ deep and $60-\mu \mathrm{m}$-wide plasma-etched grooves.

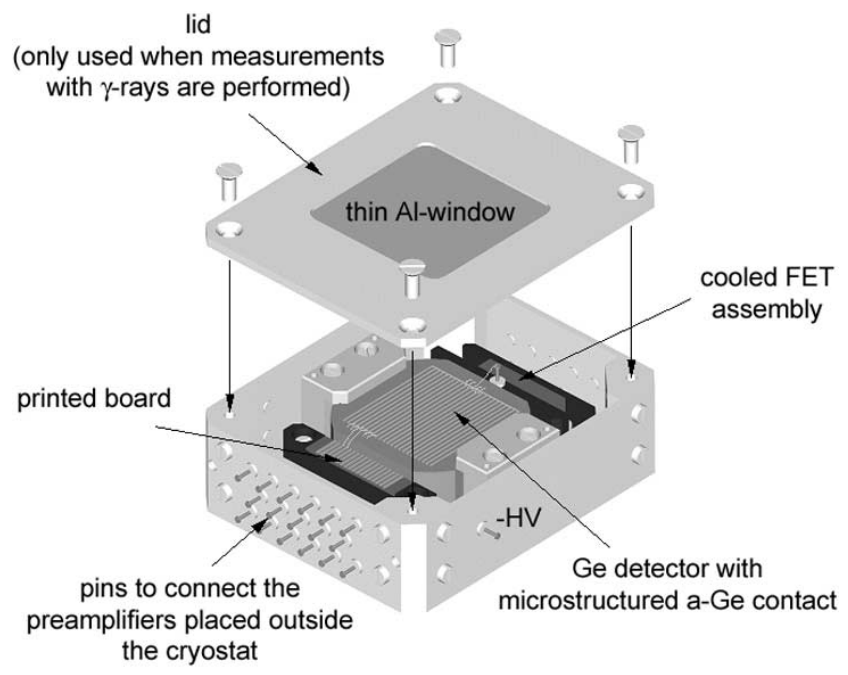

Fig. 2. Schematic view of the detector housing showing the detector and the FET assembly.

and amounted to $16 \mathrm{~mm}$. Apart from the new outline, the boron implanted $\mathrm{p}^{+}$-contact $\left(20 \mathrm{kV}, 10^{14} \mathrm{~cm}^{-2}\right)$ covered with a $0.31-\mu \mathrm{m}$-thick aluminum evaporated layer was the same as before.

A simple 50-strip structure with a pitch of $615 \mu \mathrm{m}$, surrounded by a guard-ring, was produced by means of photolithography and subsequent plasma etching of grooves through the a-Ge contact [1]. The shape of the 33-mm-long grooves and the cross section of the structured a-Ge contact are shown in Fig. 1.

The detector was mounted in the housing shown in Fig. 2. The negative bias voltage can be applied on the unstructured $\mathrm{p}^{+}$-contact (isolated from the ground). The guard-ring of the structured a-Ge contact is grounded through the two metal clamps which fasten the detector in the housing. Several strips can be connected to the printed board. One of the strips in the middle of the structure can be connected by bonding to the FET, which is placed close to the detector together with the feedback resistor and capacitor in the original Canberra assembly (first stage of the Canberra preamplifier, Model 2002). The neighboring strips can be connected by bonding to the grounded guard-ring. An influence of infrared radiation on the detector leakage current can be excluded by covering the housing with the lid.

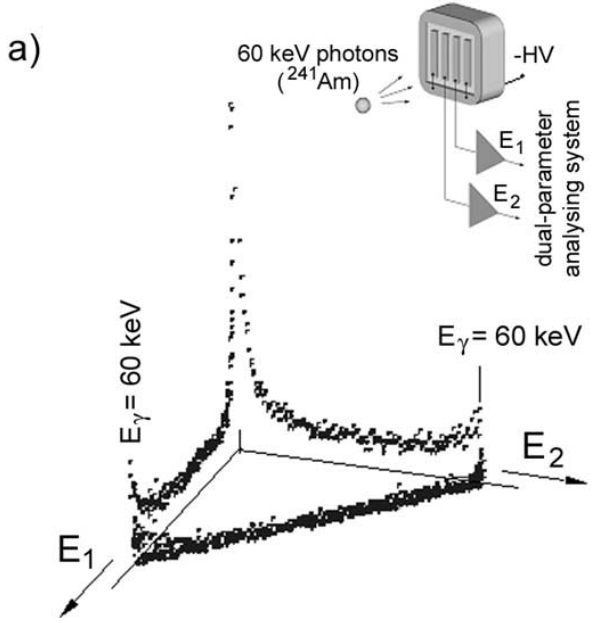

b)

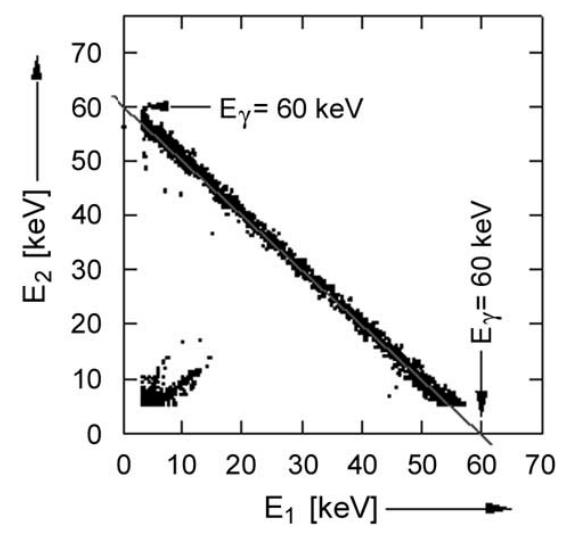

Fig. 3. Measurement of the charge splitting between two adjacent strips. (a) Two parameter energy spectrum (3-D presentation) showing single events detected with the energies $E_{1}$ or $E_{2}$ and coincident events presented on the straight band between the two 60- keV peaks. (b) The same spectrum, but in 2-D presentation.

\section{MEASUREMENTS}

\section{A. Expected Results}

1) Charge Splitting Between Two Adjacent Strips Measured With 60-keV Photons Impinging on the Structured a-Ge Contact: For the investigation of the charge splitting between the adjacent strips, each of them was connected to an amplifier chain. At the bias voltage of $-1500 \mathrm{~V}(\sim 500 \mathrm{~V}$ more than the depletion voltage), the output signals $\mathrm{E}_{1}$ and $\mathrm{E}_{2}$ generated by a ${ }^{241} \mathrm{Am} \gamma$-source were shaped with a time constant of $2 \mu \mathrm{s}$ and fed to a dual parameter analysing system (Fig. 3). The events registered simultaneously in the adjacent strips are spread between the axes $E_{1}$ and $E_{2}$. Especially from Fig. 3(b), one can conclude that practically no measurable charge losses were caused by the existence of the groove between these strips. All points on the straight band which connects the two $60-\mathrm{keV}$ peaks represent events coincidently detected in the adjacent strips with the summed energy of $60 \mathrm{keV}$. Such a result was already supposed in [1].

2) Charge Splitting Between Two Adjacent Strips Measured With 5.8-MeV $\alpha$-Particles Impinging on the Unstructured $p^{+}$-Contact: The same measuring setup as before was used to study the charge splitting, but with $\alpha$-particles hitting the unstructured $\mathrm{p}^{+}$-contact. As in the case of $60-\mathrm{keV}$ photons irradiating the strips on the a-Ge contact, there were no indications 

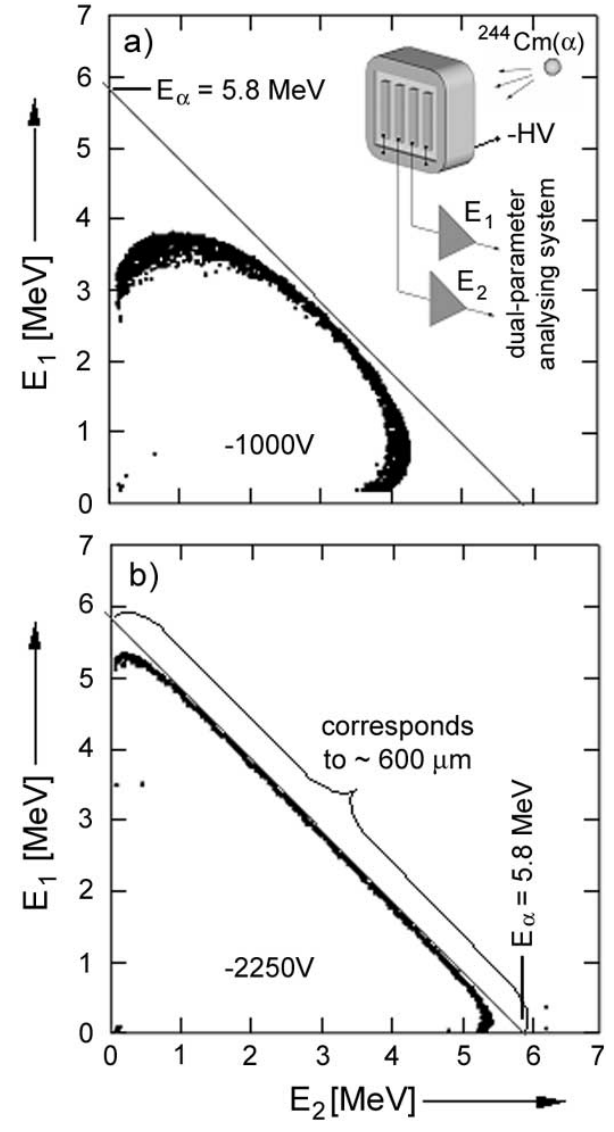

Fig. 4. Measurement of the charge splitting between two adjacent strips for $\alpha$-particles hitting the unstructured $\mathrm{p}^{+}$-contact at two different bias voltages in 2-D presentation. (a) $-1000 \mathrm{~V}$. (b) $-2250 \mathrm{~V}$ in 2-D presentation.

of any charge losses caused by the existence of the groove. This conclusion is based on the result of the charge sharing showed in Fig. 4. For the bias voltage of $-1000 \mathrm{~V}(\sim$ equal to the depletion voltage), only a small part of the coincident events gives the full energy of the $\alpha$-particles, as shown in Fig. 4(a). From this result, one can conclude that the electrons generated by the $\alpha$-particles have been laterally spread on their way to the a-Ge contact forming a cloud about $1200 \mu \mathrm{m}$ in width. Furthermore, the maximum energy registered in either of the strips does not exceed $\sim 70 \%$ of the particle energy.

Even after increasing the bias voltage to $-2250 \mathrm{~V}$, not more than $\sim 90 \%$ of the particle energy can be registered in either of the strips, as shown in Fig. 4(b). But in this case, the full energy of the $\alpha$-particles is registered in about $90 \%$ of the coincidental events. Therefore, the width of the electron cloud collected on the a-Ge contact for this bias must be more than $615 \mu \mathrm{m}$.

In addition, excellent position information for $\alpha$-particles hitting the unstructured $\mathrm{p}^{+}$-contact can be extracted from the 2-D spectrum shown in Fig. 4(b). A straight line connecting the $\alpha$-peaks on the axes $E_{1}$ and $E_{2}$ can be achieved by a careful choice of the three parameters: the thickness, the pitch and the bias voltage of the detector. Each point on this straight line will represent an impact position of the $\alpha$-particles on the rear contact. The distance between the two $\alpha$-peaks corresponds to the pitch of the structure $(615 \mu \mathrm{m})$. Assuming that $\sim 5 \%$ of the distance can be easily determined, a position resolution of $\sim 30 \mu \mathrm{m}$ should be achievable. Similar considerations can also be applied for low-energy photons, electrons, and ions.

3) Energy Resolution: An important aspect concerning possible applications of position-sensitive germanium detectors with grooved structures is the practically achievable energy resolution for a position element. The resolution figures will strongly depend on the length and the cross section of the grooves which surround the position element, i.e., on two variables which determine the capacitance of the position element to the neighboring elements.

In order to measure the energy resolution, one of the strips in the middle of the structure (Fig. 2) was connected by bonding to the encapsulated FET placed close to the detector together with the feedback capacitor and resistor in the original Canberra assembly (first stage of the Canberra 2002 preamplifier). The neighboring strips were connected by bonding to the grounded guard-ring. The influence of the infrared radiation on the leakage current was reduced by covering the housing with the lid. The rest of the preamplifier was placed outside the vacuum chamber. After cooling the detector setup with liquid nitrogen, a temperature of $92 \mathrm{~K}$ was achieved.

A typical energy spectrum of the low-energy photons from ${ }^{241} \mathrm{Am}$ measured at the bias voltage of $1500 \mathrm{~V}$ with an amplifier shaping time of $10 \mu \mathrm{s}$ is shown in Fig. 5. The resolution for 14-keV photons was slightly better and amounted to $0.72 \mathrm{keV}$ [FWHM]. The contribution of the preamplifier itself, subsequently measured at $92 \mathrm{~K}$ without the strip-FET connection, was $0.42 \mathrm{keV}$ [FWHM]. The resolution figures are far away from those achievable with good commercial Ge detectors for low-energy photons (for example $0.35 \mathrm{keV}$ [FWHM] at $60 \mathrm{keV}$ ). All of these results are preliminary, because the measuring setup was far from being optimized (for example, vibrations due to the vacuum pump, long distance between the FET and the preamplifier outside the vacuum chamber, encapsulated FET, problems with the ground, ....). Even better energy resolutions will probably be possible. Apart from the improvements of the measuring setup, there are also certain chances to reduce the interstrip capacitance through a proper groove form (deeper and/or wider grooves).

\section{B. Unexpected Results: Charge Splitting Between Two Adjacent Strips Measured With 5.8-MeV $\alpha$-Particles Impinging on the Structured a-Ge Contact}

Surprising results were obtained for $\alpha$-particles impinging on the structured a-Ge contact, as shown in Fig. 6. No coincident signals were detected on the line which connects the two $\alpha$-peaks [Fig. 6(a)]. At the same time the detector was irradiated with 661-keV photons from ${ }^{137}$ Cs. As shown in Fig. 6(b), "normal" coincident signals generated by fully absorbed photons were spread on the line that connects the two $\gamma$-peaks, indicating no charge losses caused by the groove between the two strips.

For $\alpha$-particles, however, each of the preamplifiers connected to the two strips delivered pulses of both polarities. The pulses having proper positive polarity ranged up to the level of $110 \mathrm{mV}$, whereas the pulses of "abnormal" polarity (negative) reached only about half of this value. Furthermore, the intensity of the 


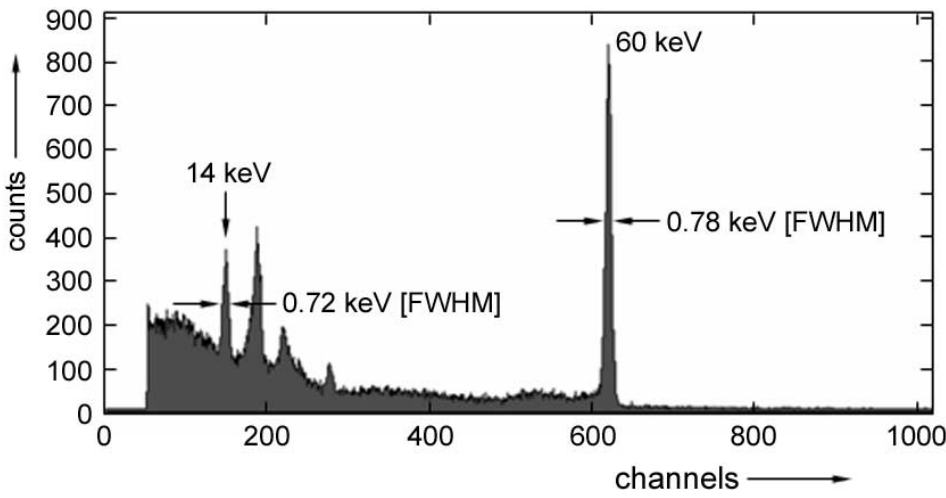

Fig. 5. Spectrum of low-energy photons from ${ }^{241} \mathrm{Am}$ measured with a 33-mm-long and 555- $\mu \mathrm{m}$-wide strip connected to a closely placed FET.

a)

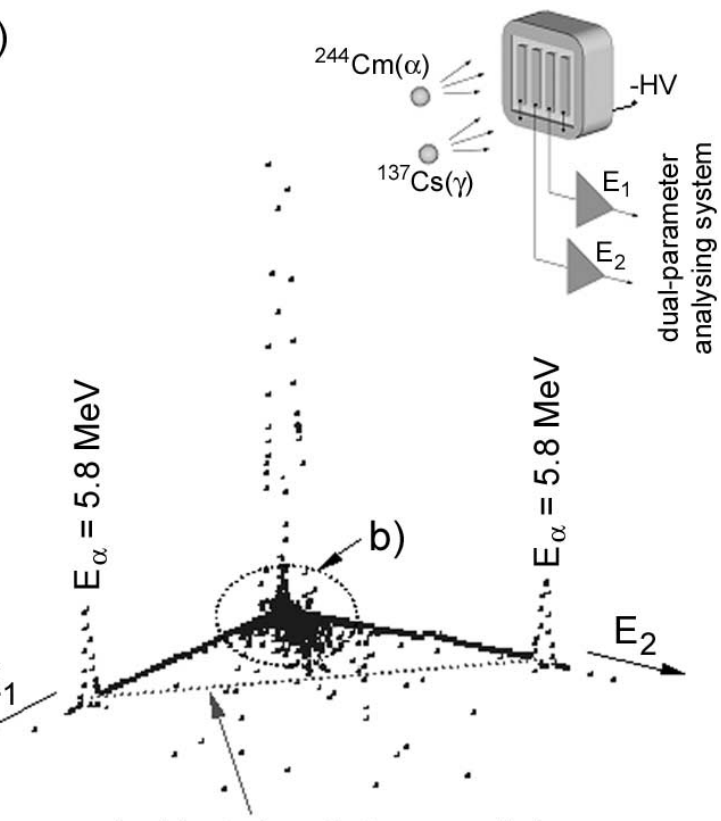

no coincident signals for $\alpha$-particles

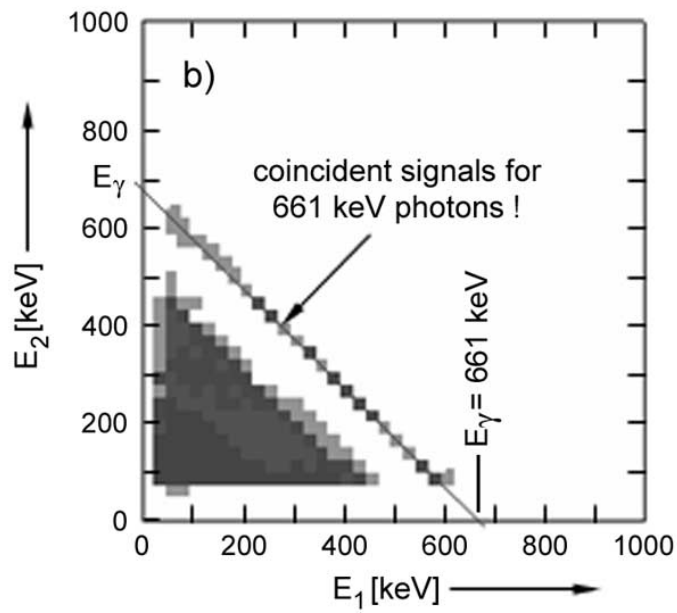

Fig. 6. Measurement of the charge splitting between two adjacent strips simultaneously irradiated with $5.8-\mathrm{MeV} \alpha$-particles $\left({ }^{244} \mathrm{Cm}\right)$ and $661-\mathrm{keV}$ photons $\left({ }^{137} \mathrm{Cs}\right)$. (a) Two parameter energy spectrum in 3-D presentation. (b) Enlarged low-energy part of the spectrum shown above, but in 2-D presentation. The gamma rays were added to show that: 1) the measurement system was functioning properly; and 2) the charge collection was complete for events generated by photons.

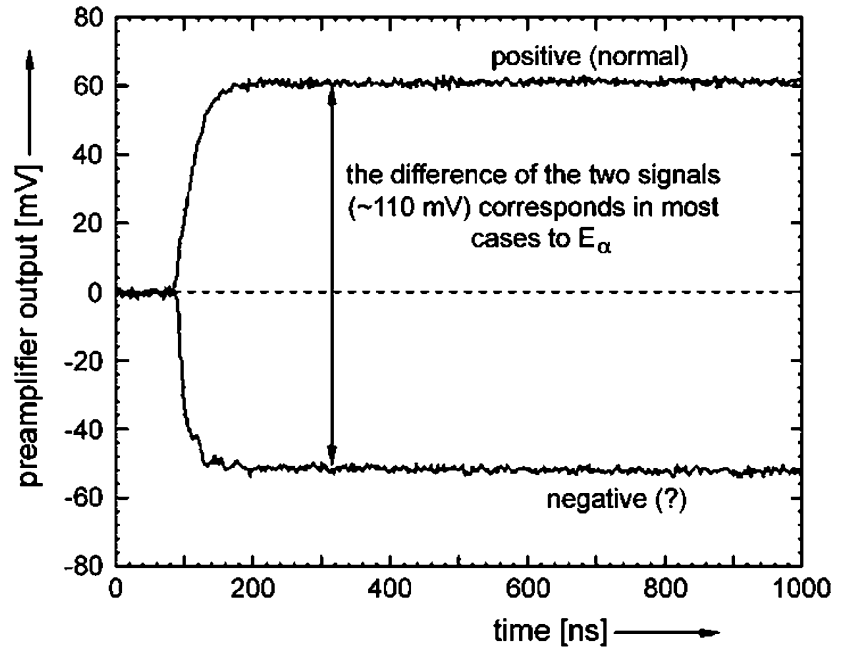

Fig. 7. Coincident preamplifier signals from two adjacent strips generated by 5.8-MeV $\alpha$-particles impinging on the structured a-Ge contact.

positive pulses was higher than that of the negative ones. But the most interesting point is that normally positive signals from one of the strips occasionally coincided with "abnormal" negative signals from the other strip and vice versa, as illustrated in Fig. 7. In most cases, the difference of the pulse heights corresponded to the energy of the $\alpha$-particles.

Additional information can be extracted from the spectra taken with the dual-parameter analysing system shown in Fig. 8. Coincidentally registered "normal" $\mathrm{E}_{1}$-pulses and inverted "abnormal" $E_{2}$-pulses are shown in Fig. 8(a). "Normal" $\mathrm{E}_{2}$-pulses in coincidence with inverted "abnormal" $\mathrm{E}_{1}$-pulses are represented in Fig. 8(b).

- Inverted signals are always not higher than $\mathrm{E}_{\alpha} / 2$.

- The majority of coincidently registered "normal" and inverted "abnormal" signals practically deliver the full energy of $\alpha$-particles.

- Smaller part of the coincident signals have about the same amplitudes.

Unfortunately, we have not been able to explain these effects. Even more, one cannot state how large is the region from which these "abnormal" pulses were coming. The measurements with 60-keV photons shown in Fig. 3 suggest only that this region 

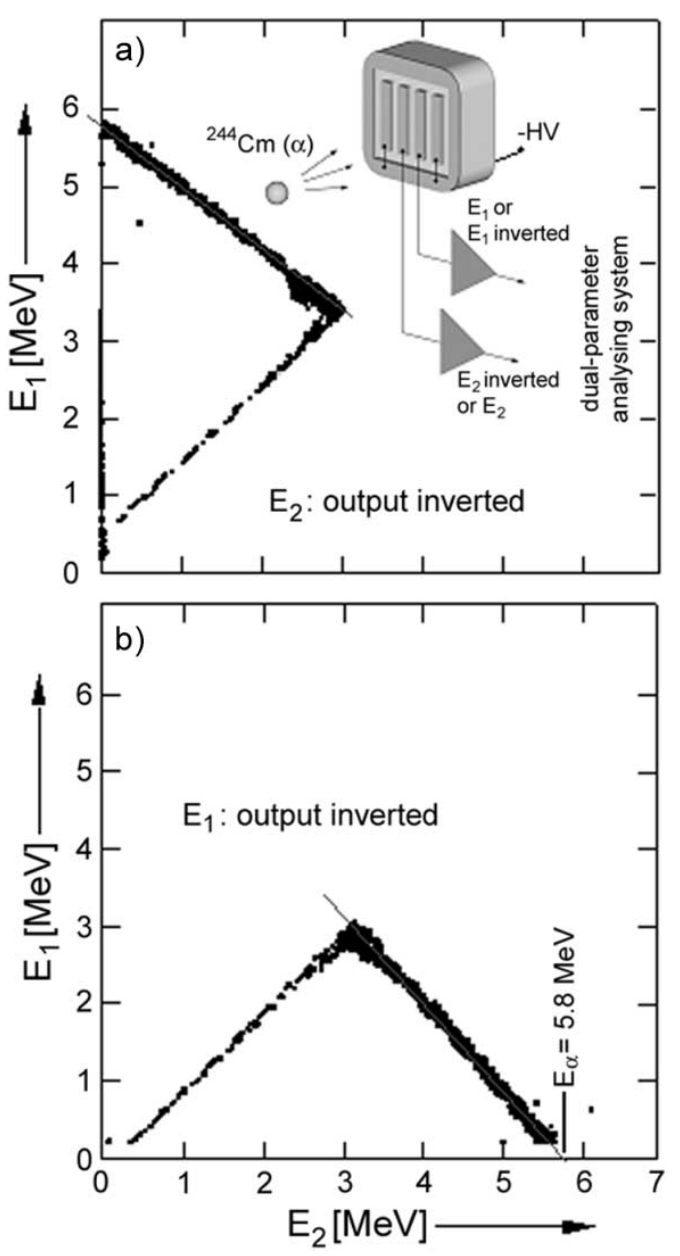

Fig. 8. Coincident signals from two adjacent strips generated by $5.8-\mathrm{MeV}$ $\alpha$-particles impinging on the structured a-Ge contact. (a) "Normal" $E_{1}$ output combined with the inverted $\mathrm{E}_{2}$ output. (b) "Normal" $\mathrm{E}_{2}$ output combined with the inverted $\mathrm{E}_{1}$ output.

should be quite small. A scan with a small collimated photon beam from one to the other strip could reveal the size of this region.

\section{CONCLUSION AND OUTLOOK}

As expected, the measurements of the charge splitting between two adjacent strips on the structured a-Ge contact with the aid of $60-\mathrm{keV}$ photons irradiating this contact confirmed that practically no measurable charge losses were caused by the existence of the groove between these strips. But the even stronger proof with $\alpha$-particles impinging on the structured a-Ge contact revealed serious problems concerning charge collection in a probably very small region just below the groove. Further investigations should be performed to find the cause for the inverted signals and the size of the region of abnormal charge collection. The same measurements as above should also be performed for structured a-Ge contacts on p-type germanium.

Very accurate position measurements of $\alpha$-particles hitting the contact opposite to the strips could be performed through proper adjustment of the bias voltage. Position accuracies amounted to about $5 \%$ of the pitch are achievable. An interesting avenue for further work would exploit the charge splitting for short range charged particles or low energy photons hitting the contact opposite to the strips just under readout to achieve the best possible position resolution using a relatively small number of readout chains.

Some progress is achievable concerning the improvement in energy resolution, especially with regard to the interstrip capacitance. Deeper and wider grooves will reduce the contributions of capacitance and dielectric noise to the energy resolution. In addition, the readout of the position elements by means of chip electronics should be a further step in the development of position-sensitive germanium detectors.

\section{ACKNOWLEDGMENT}

The authors gratefully acknowledge the technical support given by G. Fiori and H. Metz, members of the Laboratory for Semiconductor Detectors at the Nuclear Physics Institute.

\section{REFERENCES}

[1] D. Protić and T. Krings, "Microstructures on Ge detectors with amorphous Ge contacts," IEEE Trans. Nucl. Sci., vol. 50, pp. 998-1000, Aug. 2003.

[2] G. Riepe and D. Protić, "Thick silicon strip detectors," Nucl. Instrum. Methods, vol. 226, pp. 103-106, 1984.

[3] D. Protić and G. Riepe, "Position-sensitive germanium detectors," IEEE Trans. Nucl. Sci., vol. NS-32, pp. 553-555, Feb. 1985.

[4] D. Protić, T. Krings, and R. Schleichert, "Development of double-sided microstructured $\mathrm{Si}(\mathrm{Li})$ detectors," IEEE Trans. Nucl. Sci., vol. 49, pp. 1993-1998, Aug. 2002.

[5] P. N. Luke, C. P. Cork, N. W. Madden, C. S. Rossington, and M. F. Wesela, "Amorphous Ge bipolar blocking contacts on Ge detectors," IEEE Trans. Nucl. Sci., vol. 39, pp. 590-594, Aug. 1992.

[6] M. Amman and P. N. Luke, "Three-dimensional position sensing and field shaping in orthogonal-strip germanium gamma-ray detectors," Nucl. Instrum. Methods Phys. Res., vol. A452, pp. 155-166, 2000.

[7] W. Coburn, S. E. Boggs, S. Amrose, R. P. Lin, M. T. Burks, M. Amman, P. N. Luke, N. W. Madden, and E. L. Hull, "Results of charge sharing tests in a Ge-strip detector," in Proc. IEEE Conf. Nuclear Science Symp., San Diego, CA, 2001.

[8] M. Burks, N. Madden, E. Hull, L. Fabris, V. Riot, and K. Ziock, "Gamma ray imagers based on 3-D position sensitive germanium strip detectors," in Proc. IEEE Conf. Nuclear Science Symp., Norfolk, VA, 2002.

[9] P. N. Luke, R. H. Pehl, and F. A. Dilmanian, "A 140-element Ge detector fabricated with amorphous Ge blocking contacts," IEEE Trans. Nucl. Sci., vol. 41, pp. 976-978, 1994.

[10] M. Amman, private communication.

[11] V. Radeka, private communication.

[12] _ , "Signal processing for detectors: Selected topics," in Proc. IEEE Conf. Nuclear Science Symp., Norfolk, VA, 2002. 